\title{
Unusual structure of sunspot cycle 24
}

\author{
H. S. Ahluwalia ${ }^{1}$ \\ University of New Mexico \\ Albuquerque, NM 87113 USA \\ E-mail: hsa@unm.edu
}

Smooth sunspot numbers (SSNs) for cycle 24 increased since onset in December 2008, developing a shoulder in 2012, a plateau in 2013 and a peak in October 2014 followed by a decay phase well after the solar polar magnetic fields reversed. The reversals usually occur near SSN maximum, the polarity in northern hemisphere reversed in June 2012 and again in February 2014 while that in southern hemisphere reversed in June 2013. Present status of cycle 24 is described in terms of SSN and $10.7 \mathrm{~cm}(2800 \mathrm{MHz})$ microwave flux (F10.7) from the sun and its activity is compared to some prior cycles of preceding two centuries. SSNs for cycle 24 declined at a slightly faster rate than F10.7 starting near cycle 21 minimum. Also F10.7 peak occurred two months after SSN peak. Furthermore, there is an excess of SSNs in southern hemisphere for cycle 24 near maximum. Decay phase for both indices has now set in. The physical cause(es) for these differences are not known calling into question several assumptions about how a typical sunspot cycle may develop, thus highlighting our lack of understanding of how the solar Dynamo functions.

Keywords: Sunspots, Cycle 24, F10.7, Space Weather*

The 34th International Cosmic Ray Conference

30 July- 6 August, 2015

The Hague, The Netherlands

\section{${ }^{1}$ Speaker}




\section{Introduction}

Solar activity is represented by time series of SSN and $10.7 \mathrm{~cm}(2.8 \mathrm{GHz})$ microwave

flux (F10.7) from the sun. A sunspot is the seat of intense magnetic field brought to the surface from solar interior [Wang et al., 2005]. Monthly mean SSN for each calendar year since 1749 and yearly means are available to 1700 at $\mathrm{http}: / /$ sidc.oma.be/silso/. The sunspots form and dissolve on the solar surface leading $\sim 11$ y cycle. F10.7 have been recorded by radio telescopes near Ottawa (14 February 1947-31 May 1991) and Penticton, British Columbia (since 1 June 1991) at local noon (1700 GMT at Ottawa, 2000 GMT at Penticton). The data in solar flux unit (sfu) of $10-22 \mathrm{Wm}-2 \mathrm{~Hz}-1$ are posted at http://www.ngdc.noaa.gov/stp/solar/flux.html. F10.7 tends to follow changes in solar ultraviolet that influence the upper atmosphere and ionosphere [Lean, 1987; Foukal, 1998; Henney et al, 2012 and references therein].

\section{Data}

Figure 1 is a plot of the annual mean SSN (black) and F10.7 (sfu) in red for 1947-2013, for five complete cycles $(19-23)$ and parts of other two $(18,24)$; 11y mean is shown by thick lines in corresponding colors. The horizontal dashed line for F10.7 is drawn at $60 \mathrm{sfu}$. The following points are noted.

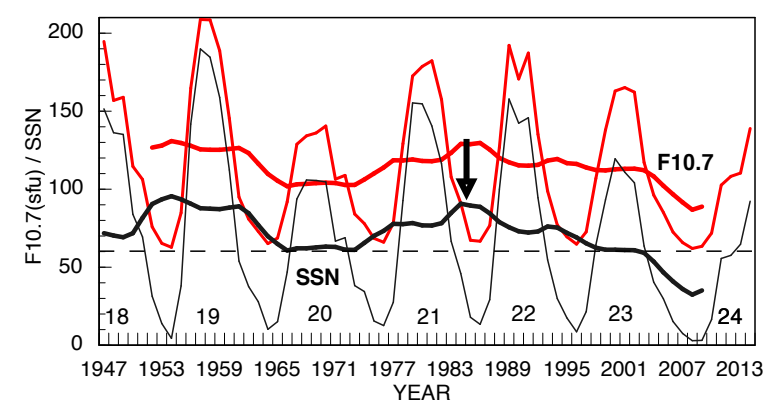

Figure 1. Yearly SSN (black), F10.7 (red) for 1947-2014; thick lines are 11y mean, arrow indicates the point of steeper descent for SSN.

1. Timelines for yearly SSN and F10.7 track well; correlation coefficient (cc) $=0.99$. Both exhibit 11y period and reach minimum in the same year.

2. SSNs rise to a peak and decrease to a variable minimum close to zero, deeper minima occur for cycles 18 and 23 .

3. F10.7 reaches minimum at the same time as SSN but at $\sim 60 \mathrm{sfu}$ for each cycle. The reason for this difference in behavior is not understood.

4. Cycle 22 has a double peak structure in both time series.

5. Cycle 24 timeline for SSN and F10.7 both indicate a plateau and a steep rise to a peak, a distinctly different profile from six previous cycles; it may be mimicking cycle 15 .

6. Decreasing trend exists for $11 \mathrm{y}$ mean starting near the minimum of cycle 21 (see arrow); SSN decline is steeper than F10.7 implying that relationship between them is drifting, calling into question the assumption that there is a close match between them. 
Yearly SSN and B are related ( $\mathrm{cc}=0.94)$ [Ahluwalia, 2013] so one should understand how F10.7 relates to solar fields [Henney et al, 2012]. Ahluwalia [2012] argues that Sun is transitioning to low activity state in a complex way leading to a Dalton like grand minimum in the twenty-first century.

\section{Unusual structure of cycle 24}

We compare growth of SSN and F10.7 for cycle 24 with cycle 15 timeline. The smoothed monthly mean SSN (black) and F10.7 (red) for cycle 24 are normalized to zero at their onset month (December 2008). Figure 2 shows growth for cycles 24, 15, F10.7 for 72 months after onset. Cycle 24 follows timeline of cycle 15 and F10.7 for 14 months, afterwards the rise of cycle 15 is steeper and F10.7 flatter. Cycle 24 develops a shoulder in February-March 2012, lingers near a plateau in 2013 and develops a delayed peak in October 2014 due to activity in southern hemisphere, well after the reversal of the polar fields; the polarity in northern hemisphere reversed in June 2012 and again in February 2014 while that in southern hemisphere reversed in June 2013. F10.7 profile exhibits the same general features as cycle 24 but peak occurs two months later (cause unknown). Ascent of cycle 24 is nearly consistent with Ahluwalia and Jackiewicz [2012] - AJ12 hereafter - prediction but peak SSN is $\sim 25 \%$ greater, illustrating the difficulty in making SSN predictions. Note that cycle 24 mimicks the development phase of cycle 15 that reached a plateau and rose to a higher peak shown in Figure 2 , delayed peak for cycle 24 is not as conspicuous as that for cycle 15 and occurs later in timeline. The decay phase has set in for both indices. We shall track their timelines as cycle 24 continues to unfold. The effort might lead to an improved capability of providing timely, reliable information regarding electrical power transmission, satellite navigation and communications and asset management in space.

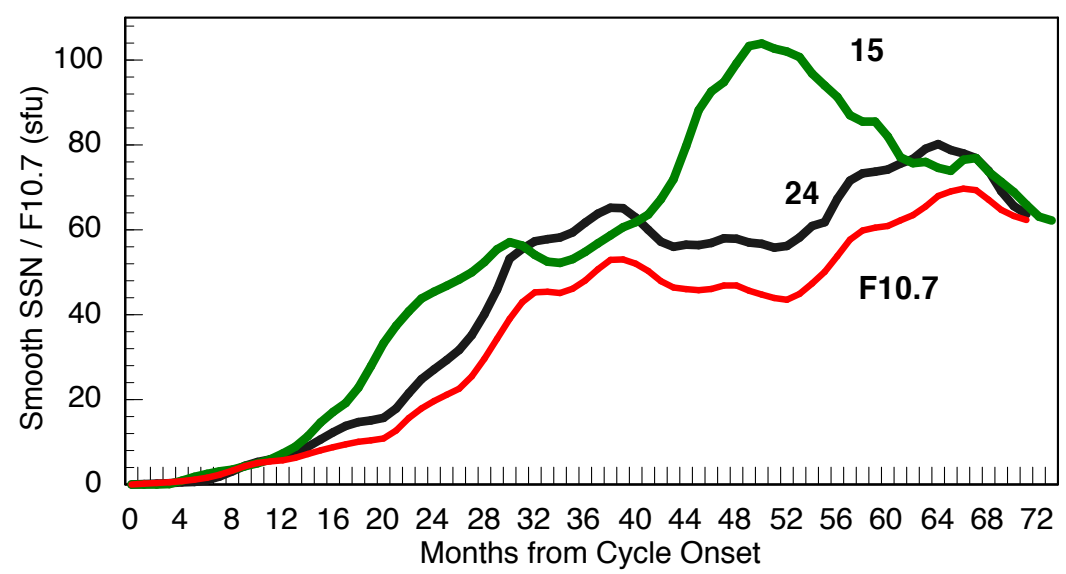

Figure 2. Growth for cycles 15 (green), 24 (black) compared with F10.7 (red) since onset.

\section{Sunspot cycle 25}

Ahluwalia [2000. 2012] discovered three-cycle-quasi-periodicity (TCQP) in SSN cycles. If it endures, AJ12 predict that next two cycles may be successively less active than cycle 24; a similar conclusion is reached by: Kane, 2002; Clilverd et al., 2003, 2006; Bonev et al., 2004; Livingston and Penn, 2009; Abdussamatov, 2012; Solheim et al., 2012, Smith et al., 2014; some predict that minimum will be more like the Maunder (1645-1715) grand minimum (MM) when 
sun was almost spotless. Zolotova and Ponyavin [2015] argue that MM is not as grand as it seemed to be due to systemic underestimate of SSNs reported by different observers. According to them, "The MM looks like an ordinary secular minimum with a depressed $11 \mathrm{yr}$ solar cyclicity." Key parameters (Rm, Tr) for future cycles cannot be predicted yet but we may speculate about cycle 25 from historical perspective. Figure 3 compares SSN growth for cycle 24 with cycles in DM starting with cycle 4 for 72 months after onset. Following pattern is noted.

1. Cycles 5, 6, 7 are significantly less active than cycle 4; they define DM (1790-1830), Mt Tambora volcanic eruption (1815) happened in cycle 5 [Wood, 2014\}. Cycle 6 is less active than cycle 5 and cycle 7 is more active than cycles 5 and 6 , leading out of DM, it has a long (>12y) duration; see Table 3.3 (p. 73) in Priest (1995).

2. Cycle 14 defines the Gliesburg minimum (GM), it is significantly more active than cycles 5, 6, and 7 indicating that GM is not a grand minimum. Steinhilber and Beer [2013] argue that 208y period (DeVries/Suess cycle) is most prominent in 9400 years of low noise $10 \mathrm{Be}$ cosmogenic radionuclide record from ice cores in Greenland (see their Fig. 1c, d), they ascribe its origin to solar activity variations but there is no evidence for it in SSN record of about 400 years. They argue that solar activity will decrease during the twenty-first century agreeing with AJ12 prediction from TCQP in Ap/aa and a historic perspective [Ahluwalia, 2012] that sun is headed to Dalton-like grand minimum. If cycle 25 takes us into the grand minimum, its activity is likely to be less than half that of cycle 24 and it will likely be a long cycle.

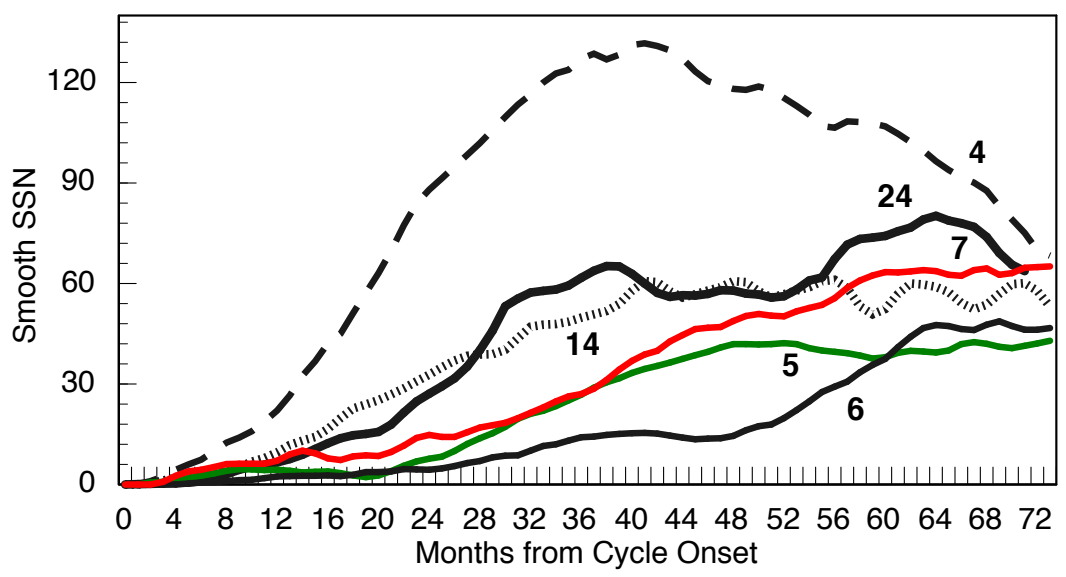

Figure 3. SSN growth for six cycles $(4,5,6,7,14$, and 24) is compared.

\section{Space weather speculations}

Since SSNs may decrease successively for the next two cycles, the value of B at $1 \mathrm{AU}$ [Ahluwalia, 2013], frequency of fast CMEs, storm sudden commencements (SSCs) and large Forbush decreases at $1 \mathrm{AU}$ shall also decrease in coming decades [Ahluwalia and Kamide, 2005; Ahluwalia et al., 2007], lower than anytime during the space age, accompanied by a significant increase in low energy GCRs [Ahluwalia and Ygbuhay, 2010; Mewaldt, 2010] and reduced ionosphere disturbances. However, one cannot rule out an occurrence of an extreme CME in future [National Research Council Report, 2008], the 1859 CME could cause a global blackout today. Clark [2007] gives a riveting account of Carrington [1859] observations and 
global havoc caused by the CME. An extreme CME occurred in July 2012, detected by STEREO satellites [Baker et al., 2013], it was directed away from the earth. Vasyliunas [2011] argues that worst case SSC cannot be more than about $40 \%$ as strong as SSC of 1859 Carrington event [Gonzalez et al., 2011]. Regrettably, space weather effects are poorly quantified for any type of space storm, particularly larger ones that might hit the earth [Schrijver and Beer, 2014].

\section{Conclusions}

The principal results of this study may be stated as follows.

1. Cycle 24 with onset in December 2008 lingered near a shoulder in 2013 and reached a peak in October 2014 well after reversals of polar fields due to activity in southern hemisphere, differing from timeline of a typical SSN cycle that rises to a peak and decreases to a variable minimum closer to zero. The decay phase of cycle 24 is underway; physical cause(es) for the conspicuous north-south asymmetry in SSNs is not understood [Shetye et al., 2015]. SSNs declined at a faster rate than F10.7 starting near cycle 21 minimum; F10.7 reached the peak two months after SSN peak for cycle 24. The physical cause(es) for these differences are not known, this calls into question the assumption (unproven) that there is a close match between the two indices.

2. Predictions for key parameters for cycle 24 [Pesnell, 2012] fell short, some were close [Schatten, 2005; Svalgaard et al., 2005; AJ12] others wide off the mark [Dikpati et al; 2006], highlighting the fact that we do not yet understand how solar Dynamo works.

3. If TCQP [Ahluwalia, 2000, 2012] endures, next two cycles $(25,26)$ shall be successively less active and phenomena that track SSNs shall be significantly affected. We are entering a new era where value of $\mathrm{B}$, frequency of fast CMEs, storm sudden commencements (SSCs) and large Forbush decreases at 1 AU shall all decrease, lower than any time during the space age, accompanied by a significant increase in low energy GCRs and reduced ionosphere disturbances. However, one cannot rule out the occurrence of an extreme $\mathrm{CME}$ in future.

\section{Acknowledgement}

I thank R.C. Ygbuhay for technical assistance.

\section{References}

[1] H.I. Abdussamatov, Bicentennial decrease of the total solar irradiance leads to unbalanced thermal budget of the earth and the little ice age (2012), Applied Phys. Res., 4, 178-184.

[2] H. S. Ahluwalia, Ap time variations and interplanetary magnetic field intensity (2000), J. Geophys. Res., 105, 27481- 27487.

[3] H.S. Ahluwalia and Y. Kamide. Gnevyshev gap, Forbush decreases, ICMEs, and solar wind electric field: Relationships (2005), Adv. Space Res., 35, 2119-2123.

[4] H.S. Ahluwalia and R. C. Ygbuhay. Current forecast for sunspot cycle 24 parameters (2010), In:Maksimovic, K. et al. (Eds.), Solar wind 12: Twelfth Int. Solar Wind Conf., AIP: CP1216, pp. 671-674. 
Unusual structure of sunspot cycle 24

[5] H.S. Ahluwalia, Three-cycle quasi-periodicity in solar, geophysical, cosmic ray data and global climate change (2012), Indian J. Radio \& Space Phys., 41, 509-519.

[6] H.S. Ahluwalia and J. Jackiewicz. Sunspot cycle 23 descent to an unusual minimum and forecasts for cycle 24 activity (2012), Adv. Space Res. 50, 662-668.

[7] H.S. Ahluwalia, Sunspot numbers, interplanetary magnetic field, and cosmic ray intensity at earth: Nexus for the twentieth century (2013), Adv. Space Res., 52, 2112-2118.

[8] D.N. Baker, X. Li, A. Pulkkinen, C. Ngwira, M.L. Mays, A.B. Galvin, and K.D.C. Simunac. A major solar eruptive event in July 2012: Defining extreme space weather scenarios (2013), Space Weather, 11, 585-591, doi:10.1002/swe.20097.

[9] B.P. Bonev, K.M. Penev, and S. Sello. Long-term variability of the solar cycle in the 21st century (2004), Astrophys. J., 605, L81-L84.

[10] R.C. Carrington, Description of a singular appearance seen in the Sun on September 1, 1859 (1859), Monthly Notices, Roy. Astron. Soc, 20, 13-.

[11] S. Clark, The Sun Kings: The unexpected tragedy of Richard Carrington and the tale of how modern astronomy began (2007), Princeton University Press, Princeton, N.J.

[12] M.A. Clilverd, E. Clarke, H. Risbeth, T.G.C. Clark, and T. Ulich. Solar activity in 2100. Astron. Geophys. (2003), 44, 20-22.

[13] M.A. Clilverd, E. Clarke, T. Ulich, and H. Risbeth. Predicting solar cycle 24 and Beyond (2006), Space Weather, 4, S09005, 7 pages, doi:10.1029/2005SW000207.

[14] M. Dikpati, Toma, G., Gilman, P.A. Predicting the strength of solar cycle 24 using a flux transport dynamo-based tool (2006). Geophys. Res. Lett., 33, L05102, doi:10.1029/2005GL025221.

[15] P. Foukal, Extension of the F10.7 index to 1905 using Mt. Wilson Ca K Spectroheliograms (1998), Geophys. Res. Lett., 25, 2909-2912.

[16] W.D. Gonzalez, E. Echer, A.L. Clua de Gonzalez, B.T. Tsurutani, and G.S. Lakhina. Extreme geomagnetic storms, recent Gleissberg cycles and space era superintense storms (2011), J. Atmos. Sol. Terr. Phys., 73, 1447-1453.

[17] C.J. Henney, W.A. Toussaint, S.M. White, and C.N. Arge. Forecasting F10.7 with solar magnetic flux transport modeling (2012), Space Weather, 10, doi:10.1029/2011SW000748

[18] R.P. Kane, Prediction of solar activity: Role of long-term variations (2002), J. Geophys. Res., 107(A7), 1113, 3 pp.,doi: 10.1029/2001JA000247.

[19] J. Lean, Solar ultraviolet irradiance variations: A review (1987), J. Geophys. Res., 92, 839-868.

[20] W. Livingston and M. Penn. Are sunspots different during this solar minimum? (2009), EOS, Trans. Am. Geophys. Union, 90(30), 257.

[21] W. Livingston, M.J. Penn, and L. Svalgaard. Decreasing sunspot magnetic fields explain unique $10.7 \mathrm{~cm}$ radio flux (2012), Astrophys. J. Lett., 757, L8.

[22] R.A. Mewaldt, Davis, A.J., Lave, K.A., Leske, R.A., Stone, E.C., Wiedenbeck, M.E., et al. (2010), Record setting cosmic ray intensities in 2009 and 2010. Astrophys. J. Lett. 723, L1. National Research Council, Severe space weather events: Understanding societal and economic impacts (2008), Workshop Report, Nat. Acad. Press, Washington, D.C.

[23] W.D. Pesnell, Solar cycle predictions (2012), Solar Phys. 281, 507-532.

[24] W.D. Pesnell, Predicting solar cycle 24 using a geomagnetic precursor pair (2014) Solar Phys., 289, 2317-2331. 
Unusual structure of sunspot cycle 24

[25] K. Schatten, Fair weather for solar cycle 24 (2005). Geophys. Res. Lett., 32, L21106, doi:10.1029/2005GL024363.

[26] C.J. Schrijver and J. Beer, Space weather from explosions on the sun: How bad could it be? (2014), EOS, Trans.Am. Geophys. Union, 95(24), 201-202.

[27] J. Shetye, D. Tripathi, and M. Dikpati. Observations and modeling of north-south asymmetries using a flux transport dynamo. Astrophys. J., 799:220 (11pp), 2015.

[28] C.W. Smith, K.G. McCracken, N.A. Schwadron and M.L.Goelzer, The heliospheric magnetic flux, solar wind proton flux, and cosmic ray intensity during the coming solar minimum, Space Weather (2014), 12, 499-507, doi:10.1002/2014SW001067.

[29] Jan-Erik Solheim, K. Stordahl, and O. Humlumc. The long sunspot cycle 23 predicts a significant temperature decrease in cycle 24 (2012), J. Atm. Sol.-Terr. Phys., 80, 267-284.

[30] F. Steinhilber and J. Beer. Prediction of solar activity for the next 500 years (2013), J. Geophys. Res., 118, 1861-1867, doi:10.1002/jgra.50210.

[31] L. Svalgaard, Cliver, E.W., Kamide, Y. Sunspot cycle 24: Smallest cycle in 20 years? (2005). Geophys. Res. Lett., 32, L01104, doi:10.1029/2004GL021664.

[32] V.M. Vasyliunas, The largest imaginable magnetic storm (2011), J. Atmos. Sol. Terr. Phys., 73, 1444-1446.

[33] Y.M. Wang, J.L. Lean, and N.R.Sheeley (2005). Modeling the sun's magnetic field and irradiance since 1713, Astrophys., J., 625, 522-538.

[34] G.D. Wood, Tambora: The eruption that changed the world (2014), Princeton University Press, 293 pp.

[35] N.V. Zolotova, D.I. Ponyavin (2015), The maunder minimum is not as grand as it seemed to be, Astrophys., J., 800:42 (7 pp). 